\title{
Review
}

\section{Bovine tuberculosis as a model for human tuberculosis: advantages over small animal models}

\author{
Ildiko Van Rhijn $^{\text {a,* }}$, Jacques Godfroid ${ }^{\text {b,d }}$, Anita Michel ${ }^{\mathrm{c}}$, Victor Rutten ${ }^{\mathrm{a}, \mathrm{b}}$ \\ a Department of Infectious Diseases and Immunology, Faculty of Veterinary Medicine, Utrecht University, Yalelaan 1, 3584CL Utrecht, The Netherlands \\ ${ }^{\mathrm{b}}$ Department of Veterinary Tropical Diseases, Faculty of Veterinary Science, University of Pretoria, Private Bag X04, Onderstepoort 0110, South Africa \\ c Onderstepoort Veterinary Institute, Agricultural Research Council, Private Bag X5, Onderstepoort 0110, South Africa \\ ${ }^{\mathrm{d}}$ Norwegian School of Veterinary Science, Section of Arctic Veterinary Medicine, Stakkevollveien 9010, Troms $\phi$, Norway
}

Received 15 October 2007; accepted 14 April 2008

Available online 22 April 2008

\begin{abstract}
For the development of vaccines and treatments against tuberculosis, animal models are needed. In this review, the pathogenesis and immune responses during human and bovine tuberculosis will be compared. Special attention will be paid to latency, because this feature has recently become the basis of specialized vaccines against latency antigens.
\end{abstract}

(c) 2008 Elsevier Masson SAS. All rights reserved.

Keywords: Models; Animal; Tuberculosis; Mycobacterium tuberculosis

\section{Introduction}

Tuberculosis causes enormous morbidity and mortality in humans world wide, despite the use of existing therapeutic drugs, due to the low efficacy of the currently available vaccine, BCG. Mice, guinea pigs, and rabbits experimentally infected with Mycobacterium tuberculosis are used as models for human tuberculosis. A wealth of knowledge from these models and from human tuberculosis itself is available and has led to the development of novel, experimental vaccines and drugs against human tuberculosis. It has been estimated that approximately 250 novel vaccine candidates against tuberculosis have been developed recently [1].

Bovine tuberculosis caused by Mycobacterium bovis is a disease of economical, veterinary public health, and zoonotic concern and therefore understanding the immune response during bovine tuberculosis and the development of a proper vaccine is a major aim in itself. The close relationship between M. tuberculosis and M. bovis, and the fact that primates and glires (to

\footnotetext{
* Corresponding author. Tel.: +31 30 2534608; fax: +31 302533555 .

E-mail address: i.vanrhijn@uu.nl (I. Van Rhijn).
}

which mice, guinea pigs and rabbits belong) are as related to each other as to artiodactyls (to which cattle belong), justifies the application of knowledge from small animal models and human tuberculosis to bovine tuberculosis and vice versa. However, research addressing bovine tuberculosis is regularly performed directly in the target species by veterinary scientists, and research addressing human tuberculosis is performed using mice, guinea pigs, rabbits, and monkeys as models. Unfortunately, bovine tuberculosis is not generally regarded as a model for human tuberculosis [2]. Some apparent disadvantages of bovine tuberculosis as a model for human tuberculosis as compared to the small animal models are of non-scientific nature, like the higher costs, the time consuming nature of working with a slowly progressing disease, the difficulty of housing large animals under high containment conditions. However, compared to the Rhesus monkey model, the costs, ethical disadvantages, and health risks for animal caretakers are lower. The fact that research groups in the veterinary sciences and the medical science are generally neither integrated, nor closely collaborating makes the bovine model less accessible to medical scientists than the small animal models, although recently integrated human/bovine diagnostic and vaccine studies using ESAT-6 and CFP10 have been published [3]. 
It is not clear whether a vaccine against tuberculosis should target $\gamma \delta \mathrm{T}$ cells and/or T cells restricted by MHC class I, class II, and/or CD1, and whether it should include latency antigens, and/or antigens associated with actively growing bacteria. The answers to these questions may be quite different when addressed in different species, an idea that has been put forward already by Koch in 1884 [4] by stating "In studying the appearances presented by tuberculosis in the different kinds of animals, the remarkable fact comes to light that the disease appears under a different aspect in each species". This implies that picking the right model species for studying a certain aspect of tuberculosis may be crucial. In this review, aspects of bovine tuberculosis that may provide unique opportunities as a model for human tuberculosis will be addressed. The advantages of rabbits and guinea pigs over mice are known to be their sensitivity to tuberculous bacteria, and the resemblance of their lung pathology to human lung pathology. Here we argue that cattle may even be a better model. Utilizing selected clinical and immunological aspects that show high similarities to human tuberculosis will provide invaluable opportunities for assisting basic and clinical research. The characteristics of the bovine model that will be discussed are the parallels in clinical aspects such as route of exposure, infectious dose, course of the disease, latency, pathology, and immunological aspects such as the development of CD1restricted $\mathrm{T}$ cell responses and $\gamma \delta \mathrm{T}$ cell responses.

\section{Exposure and infectious dose}

Like in humans, aerosolized bacteria are the most common source of natural infection in cattle, and the primary site of natural infection is the respiratory tract [5]. One to three bacteria can be sufficient to cause infection in cattle [6,7], a number comparable to what has been suggested to be the minimal infectious dose for humans. Initially, the minimal infectious dose gives rise to a single infectious focus in the lung, which is difficult to detect. Natural aerogenic infections are in the early stages mostly detected in the lymph nodes of the head and thoracic cavity by visual inspection or bacterial culture [8]. Later in the course of natural infection, a single primary focus may become visible in the lung [9, pp. 18-19]. Experimental infections have been performed in cattle with different infectious doses and routes of exposure. Routes of experimental exposure that result in lesions that are comparable to what is seen in natural aerogenic infections are the intratracheal, intratonsillar, and intranasal route. In general, all animals will progress into severe disease when large infectious doses are administered. At lower infectious doses, the proportion of diseased animals drops, as well as the severity of the disease. A dose of $10^{3}$ given intratracheally induces normal pathology when assessed 4-5 months after infection $[7,10,11]$. Intratonsillar exposure is commonly used because of its technical convenience in combination with the fact that it still induces lesions that are comparable to what is seen in natural infections [12]. An experimental infectious dose of $10^{3}-10^{4}$ given intranasally gives a slightly atypical pathology with more lesions than natural infections, and with a higher occurrence of infection of the nasal turbinates [13]. Two routes of exposure that do not mimic natural aerogenic infection are subcutaneous exposure, which gives rise to a necrotic tumor at the site of inoculation and subsequent infection of multiple tissues, and intravenous exposure, which also spreads to multiple tissues, though the lungs become most heavily infected [9, p. 13].

\section{Sensitivity and progression to tuberculosis}

Ten to thirty percentage of the humans who are naturally exposed to $M$. tuberculosis get infected [14]. Of the 70-90\% of humans who do not get infected upon exposure, it is thought that their innate immune systems effectively prevented infection. Unless BCG vaccinated, these people remain skin test negative because skin testing is dependent on adaptive immunity. Of all immunocompetent infected people, $10-40 \%$ is sensitive to the development of active, progressive tuberculosis [14]. Half of the tuberculosis cases develop within a year after infection, and the other half later in life. In the other $60-90 \%$ of immunocompetent, infected people, the diseases will remain latent and does not progress. In guinea pigs and in sensitive and resistant mouse models, there is less interindividual variation and all animals succumb from infection with $M$. tuberculosis. The same holds for sensitive and resistant rabbits experimentally infected with $M$. bovis [15]. In cattle, the outcome of exposure to $M$. bovis shows interindividual differences, resembling the situation in humans exposed to M. tuberculosis. Like humans, cattle can fail to become infected, which is most likely due to an effective innate immune response. In an infected herd, where it can be assumed that all animals are exposed, a maximum of $30 \%$ of the animals becomes infected, depending on the housing conditions and the level of sanitary intervention in the herd [16]. Alternatively, they can become infected and clear the infection. In this case, animals turn skin test positive and/or their blood cells release IFN $\gamma$ upon stimulation with PPD, but they show no clinical signs of disease, no visible lesions are present when slaughtered, and M. bovis cultures are negative [17]. The question to which extent animals that are effectively infected can remain clinically latent for years, will be discussed below. Another possible outcome in exposed cattle is the development of clinical tuberculosis upon infection. The disease can follow a slowly progressive/remitting course, progress fairly rapidly, meaning that visible lesions develop within two years, or become acute and fatal within months [18, p. 96, 9, p. 19]. The relative proportion of the different courses of bovine tuberculosis is difficult to estimate, but it is clear that housing conditions and sanitizing interventions play an important role.

\section{Pathology}

\subsection{Tissue distribution}

Tuberculous lesions in humans are mainly found in the lung and the associated lymph nodes. In $85 \%$ of the immunocompetent, but tuberculosis-sensitive humans, infection is limited to the lungs [2]. Tuberculosis in immunocompromised 
individuals like AIDS patients usually spreads to multiple organs. In mice, during the initial acute phase with bacterial replication, the infection disseminates to the spleen and liver. Even when infected by inhalation, tuberculosis in rabbits and guinea pigs is also evident in spleen and liver [2,4]. All mice, rabbits, and guinea pigs show progressive lung pathology, independent from whether bacterial loads are stationary or increasing [19]. After 4 weeks, the fairly high bacterial numbers will stabilize in guinea pigs, rabbits, and resistant mice, but there is slowly progressing lung pathology that will eventually result in death $[2,15,19]$. However, in cattle, like in humans, tuberculosis is in $90 \%$ of the cases a disease of the lung and the associated lymph nodes. During the very early stages, bovine tuberculosis can be detected in the lymph nodes of the head and thoracic cavity, with the retropharyngeal lymph nodes, draining the tonsils, and mediastinal lymph nodes accounting for the majority of the lesions [8]. During intermediate stages of bovine tuberculosis, one or more visible lesions become apparent in the lungs. Other locations of tuberculous lesions are rare in cattle, but there are reports of udder infections and visceral infections, and a rare miliary form in advanced tuberculosis in old animals [5,18, pp. 99-103].

\subsection{Granulomas}

Human granulomas are highly organized structures and can become fibrotic, calcify, or evolve into cavities, all of which are present during active tuberculosis. The mouse produces a less organized, homogeneous type of lesion that barely shows necrosis, while caseous necrosis and cavitations have not been observed. Guinea pigs and rabbits form granulomas that resemble early human granulomas. Small cavities form in guinea pigs that are infected by inhalation, but the animals usually die before the cavities become larger or liquefy [9, p. 132]. Caseation also takes place in rabbits [9, p. 139]. Like in humans, heterogeneity in granuloma type is commonly found during bovine tuberculosis, and is thought to reflect differences in age of the granulomas, implying that primary foci have been developing for quite some time before infecting other parts of the lung. Liquefaction and cavitation have been described in advanced cases of bovine tuberculosis [5]. Nowadays, these features are seen less often because diseased animals are detected and culled in relatively early stages of disease, which also holds for experimentally infected animals.

\section{Latency}

Since latency is an important aspect in the pathogenesis of human tuberculosis, with implications for diagnostic procedures and novel vaccination strategies, it is a highly relevant feature in animal models as well. Clinically, latency is defined as the persistence of a tuberculous lesion with viable mycobacteria in a host without symptoms [20]. Unfortunately, proper methods for diagnosis of latent tuberculosis that also allow to distinguish it from chronic tuberculosis do not exist for humans and animals. Latency is the product of interaction between host and mycobacterium. Even though a stable or slowly progressing bacterial load can be maintained for some time in mice, rabbits, and guinea pigs, it is important to know to which extent this situation resembles human latent infection. Currently, post exposure vaccines against $M$. tuberculosis antigens that are up regulated during latency in vivo are being developed [21]. Both the clinical aspects of latency in an animal model of tuberculosis and the antigenic status of the mycobacterium during latency have to be taken into consideration when considering an animal mode for testing such vaccines.

In vitro, tuberculoid mycobacteria can go into a dormant state [22], while a non-tuberculous mycobacterium like Mycobacterium smegmatis cannot [23]. The dormant state of $M$. tuberculosis closely resembles the $M$. bovis BCG dormant state, and both are biochemically and morphologically distinct from their respective growing states [23], and it is thought that that holds for $M$. bovis wild type as well. Genes that are expressed during the dormant state of both $M$. tuberculosis and $M$. bovis BCG are: $h s p X$ (encoding $\alpha$-crystallin) [23,24], NarGHIJ (encoding nitrate reductase) [25], $g c v B$ (encoding glycine dehydrogenase) and [24], sigF (encoding a stressresponse transcription factor) [26]. From the above it becomes clear that in vitro, M. bovis and M. tuberculosis can go into a similar dormant state. The key question for testing vaccines against latency antigens is whether they do in vivo. In humans, both M. tuberculosis and M. bovis [27] can give rise to a clinically latent form that can be reactivated decades after the initial exposure. It is not clear whether mycobacteria in the Cornell model, an antibiotics-induced model for latency in mice, go into a true dormant state [28,29]. After treatment, bacteria cannot be detected in these mice, but reappear after a certain amount of time.

In vivo, cavitation is the condition that is thought to cause bacteria to go into the metabolically and morphologically distinct dormant state. Caseous tubercles that lead to cavitation do form in cattle. It has not been formally proven that in cattle bacteria go into a true dormant state, but, contrary to other animal models, this important pathological requirement is met. Rabbits and guinea pigs form cavitations, but they show progressive disease in multiple tissues, and do not go through a period of clinical latency. There are suggestions from epidemiological studies that clinical latency does occur in cattle in natural and experimental situations. Clinical latency has been suspected in animals that, upon natural or experimental exposure, turn positive in an IFN $\gamma$ release assay, may or may not turn positive in a skin test, but are without clinical signs of disease and visible lesions when slaughtered, and with negative $M$. bovis cultures [17] or positive cultures from lymph nodes only [30]. A single, primary focus is known to be able to remain latent for years, or progress only slightly [18, pp. 95-96] . This type of single, primary lung lesion in cattle, even if not progressing for many years, has long been thought to be "open" in most cases, and infectious for other animals [18, pp. 95-96, 9, p. 19]. However, the original observations leading to this conclusion may have been made in the presence of other infectious sources, and may not hold. A more recent observation that suggests the existence 
of latency is the following: typically, 10 years of skin testing and culling is needed to get rid of tuberculosis in an infected herd, even in the absence of external sources of infection, like in Australia [8,31]. Under these circumstances it appears that previously undetected infected animals can be identified many years after a testing and culling procedure, suggestive of the presence of latently infected animals that have been negative in skin tests. In three well documented Australian herds the interval between the last tuberculin reactor removed from the herd and the detection of a new reactor was over 12 years [31]. However, methods for experimental induction of latent tuberculosis in cattle do not yet exist. So, even though cattle may be the species in which latency resembles human latency optimally, there is a long road to go before a bovine model of latent tuberculosis is available, for example for testing post exposure vaccines against latency antigens. Research aiming at the development of a bovine model of latent tuberculosis may include the use of mycobacteria that are attenuated in cattle, like M. tuberculosis [32,33], or experimental treatment of $M$. bovis-infected animals with antibiotics in a way that is comparable to the Cornell model in mice $[28,29]$.

\section{Immune responses to mycobacteria in different species}

The range of reagents available for immunological research in cattle is smaller than for humans and mice, but there is a reasonable choice of lineage markers and anti cytokine antibodies, especially when compared to rabbit and guinea pig. Conventional $\mathrm{T}$ cells restricted by MHC class II and class I are thought to perform functions that are broadly conserved across the animal kingdom. CD4+ $\mathrm{T}$ cells restricted by MHC class II are known to play an important role during tuberculosis. Interestingly, some of the MHC class II-presented antigens that are currently under investigation for use in vaccines and diagnostics in human tuberculosis are also relevant during bovine tuberculosis. ESAT- 6 and CFP10 are recognized by $\mathrm{T}$ cells from tuberculous humans as well as cattle [3], and not, or to a lesser extent, by BCG vaccinated individuals.

However, MHC class II-restricted T cells are not the only ones that should be considered in the immune response against tuberculous mycobacteria. CD1-restricted T cells and $\gamma \delta \mathrm{T}$ cells are also known to be able to recognize mycobacterial antigens and are known to become activated during human tuberculosis. Even though CD1 and the $\gamma \delta$ TCR are usually considered to belong to an "innate-like" part of the immune system, and very old at an evolutionary time scale, they are less conserved across species than usually assumed. The total number of $C D 1$ genes and the isoforms present in different species varies highly, as well as the numbers and the level of homology of the segments in the $\gamma$ and $\delta$ loci that encode the $\gamma \delta$ TCR. Mice differ fundamentally from humans with regard to $\gamma \delta$ T cells and CD1, which makes them less suitable for tuberculosis research with respect to these fields.

CD1 molecules present lipid antigens to T cells. Most mycobacterial antigens that are known to be presented by CD1 are presented by group $1 \mathrm{CD} 1$ (CD1a, CD1b, and CD1c), which are absent in mice. The known CD1-presented mycobacterial antigens are diverse and include mycobacterial mycolates, diacylglycerols (LAM and PIM), polyisoprenoid lipids, sulfotrehalose-containing lipids and lipopeptides [34]. Cattle express CD1a and three CD1b isoforms, but lacks CD1c [35]. T cells from cattle infected with mycobacteria, but not from uninfected cattle, react to stimulation with mycobacterial lipids (Van Rhijn, unpublished), a situation that is comparable to human tuberculosis [36]. This makes bovine tuberculosis a suitable model to study the function of group 1 CD1-restricted T cells during tuberculosis.

Also with respect to $\gamma \delta \mathrm{T}$ cells, there are considerable differences between species. Cattle, especially young animals, have much higher percentages of circulating $\gamma \delta \mathrm{T}$ cells than humans, mice, rabbits, and guinea pig. It is clear that $\gamma \delta \mathrm{T}$ cells become activated during human tuberculosis [37] and bovine tuberculosis $[38,39]$, but it is unknown whether this happens directly via the $\mathrm{T}$ cell receptor, or by indirect mechanisms. It is unknown whether $\gamma \delta \mathrm{T}$ cells contribute to effective immunity to mycobacteria. In terms of antigen specificity, cattle have an important advantage over other animal models. A significant fraction of the $\gamma \delta \mathrm{T}$ cell population in humans and non-human primates recognizes (myco)bacterial phosphoantigens. Recognition of these antigens is $\gamma \delta \mathrm{T}$ cell receptor mediated. Phosphoantigen specific $\mathrm{T}$ cells are absent in mice, but have been reported in cattle [40]. In cattle, the question whether $\gamma \delta \mathrm{T}$ cells contribute to immunity against tuberculosis has been addressed by depleting all $\gamma \delta \mathrm{T}$ cells from an M. bovis-infected animal, which resulted in no obvious differences in the course of the disease [41]. However, others have shown that in vitro, $\gamma \delta \mathrm{T}$ cells contribute to responses against antigens present in $M$. bovis [40]. It is unknown whether there is a $\gamma \delta \mathrm{T}$ cell subset in guinea pigs and rabbits that recognizes phosphoantigens. Recently, it was found that these $\gamma \delta \mathrm{T}$ cells form $\mathrm{T}$ cell memory in primates, and may thus be good targets for vaccine development [42].

\section{Conclusions}

The use of animal models has been crucial in the characterization of host and bacterial factors that determine the immune response against tuberculous bacteria, and is expected to become even more important in the testing of novel vaccines against tuberculosis, including subunit vaccines and vaccines against latency antigens. Bovine tuberculosis may prove to be an excellent model for human tuberculosis when aspects like pathogenesis, pathology, the development of a latent state of the infection, and immune responses to tuberculosis like innate immunity, group $1 \mathrm{CD} 1$-restricted $\mathrm{T}$ cell responses, and phosphoantigen-reactive $\gamma \delta \mathrm{T}$ cells are considered.

\section{Acknowledgements}

This work was supported by the Wellcome Trust [GR078283]. 


\section{References}

[1] U. Fruth, D. Young, Prospects for new TB vaccines: stop TB working group on TB vaccine development, Int. J. Tuberc. Lung Dis. 8 (2004) 151-155.

[2] R.J. North, Y.J. Jung, Immunity to tuberculosis, Annu. Rev. Immunol. 22 (2004) 599-623.

[3] L.A. van Pinxteren, P. Ravn, E.M. Agger, J. Pollock, P. Andersen, Diagnosis of tuberculosis based on the two specific antigens ESAT-6 and CFP10, Clin. Diagn. Lab. Immunol. 7 (2000) 155-160.

[4] R. Koch, Etiology of tuberculosis, Mittheilungen aus dem Gesundheitsamte ii (1884) 65-201.

[5] E.M. Medlar, Pulmonary tuberculosis in cattle, Am. Rev. Tuberc. 41 (1940) 283-306.

[6] S.D. Neill, J.J. O'Brien, J. Hanna, A mathematical model for Mycobacterium bovis excretion from tuberculous cattle, Vet. Microbiol. 28 (1991) $103-109$.

[7] G.S. Dean, S.G. Rhodes, M. Coad, A.O. Whelan, P.J. Cockle, D.J. Clifford, R.G. Hewinson, H.M. Vordermeier, Minimum infective dose of Mycobacterium bovis in cattle, Infect. Immun. 73 (2005) 6467-6471.

[8] L.A. Corner, Post mortem diagnosis of Mycobacterium bovis infection in cattle, Vet. Microbiol. 40 (1994) 53-63.

[9] J. Francis, Tuberculosis in Animals and Man, Cassell and Company Limited, London, 1958.

[10] B.M. Buddle, J.M. Pollock, M.A. Skinner, D.N. Wedlock, Development of vaccines to control bovine tuberculosis in cattle and relationship to vaccine development for other intracellular pathogens, Int. J. Parasitol. 33 (2003) 555-566.

[11] B.M. Buddle, G.W. de Lisle, A. Pfeffer, F.E. Aldwell, Immunological responses and protection against Mycobacterium bovis in calves vaccinated with a low dose of BCG, Vaccine 13 (1995) 1123-1130.

[12] M.V. Palmer, D.L. Whipple, J.C. Rhyan, C.A. Bolin, D.A. Saari, Granuloma development in cattle after intratonsilar inoculation with Mycobacterium bovis, Am. J. Vet. Res. 60 (1999) 310-315.

[13] S.D. Neill, J.J. O'Brien, R.M. McCracken, Mycobacterium bovis in the anterior respiratory tracts in the heads of tuberculin-reacting cattle, Vet. Rec. 122 (1988) 184-186.

[14] Y.C. Manabe, W.R. Bishai, Latent Mycobacterium tuberculosis - persistence, patience, and winning by waiting, Nat. Med. 6 (2000) 1327-1329.

[15] M.B. Lurie, Experimental epidemiology of tuberculosis, J. Exp. Med. 79 (1944) 573-589.

[16] C.J. Phillips, C.R. Foster, P.A. Morris, R. Teverson, The transmission of Mycobacterium bovis infection to cattle, Res. Vet. Sci. 74 (2003) 1-15.

[17] J.M. Pollock, R.M. Girvin, K.A. Lightbody, R.A. Clements, S.D. Neill, B.M. Buddle, P. Andersen, Assessment of defined antigens for the diagnosis of bovine tuberculosis in skin test-reactor cattle, Vet. Rec. 146 (2000) 659-665.

[18] J. Francis, Bovine Tuberculosis, Staples Press Unlimited, London, 1947.

[19] D.W. Smith, G.E. Harding, Animal model of human disease. Pulmonary tuberculosis. Animal model: experimental airborne tuberculosis in the guinea pig, Am. J. Pathol. 89 (1977) 273-276.

[20] J.E. Gomez, J.D. McKinney, M. tuberculosis persistence, latency, and drug tolerance, Tuberculosis (Edinb) 84 (2004) 29-44.

[21] P. Andersen, Vaccine strategies against latent tuberculosis infection, Trends Microbiol. 15 (2007) 7-13.

[22] L.G. Wayne, C.D. Sohaskey, Nonreplicating persistence of Mycobacterium tuberculosis, Annu. Rev. Microbiol. 55 (2001) 139-163.

[23] A.F. Cunningham, C.L. Spreadbury, Mycobacterial stationary phase induced by low oxygen tension: cell wall thickening and localization of the 16-kilodalton alpha-crystallin homolog, J. Bacteriol. 180 (1998) 801-808.

[24] A. Lim, M. Eleuterio, B. Hutter, B. Murugasu-Oei, T. Dick, Oxygen depletion-induced dormancy in Mycobacterium bovis BCG, J. Bacteriol. 181 (1999) 2252-2256.
[25] I. Weber, C. Fritz, S. Ruttkowski, A. Kreft, F.C. Bange, Anaerobic nitrate reductase (narGHJI) activity of Mycobacterium bovis BCG in vitro and its contribution to virulence in immunodeficient mice, Mol. Microbiol. 35 (2000) 1017-1025.

[26] Y.C. Manabe, J.M. Chen, C.G. Ko, P. Chen, W.R. Bishai, Conditional sigma factor expression, using the inducible acetamidase promoter, reveals that the Mycobacterium tuberculosis sigF gene modulates expression of the 16-kilodalton alpha-crystallin homologue, J. Bacteriol. 181 (1999) 7629-7633.

[27] A. Fritsche, R. Engel, D. Buhl, J.P. Zellweger, Mycobacterium bovis tuberculosis: from animal to man and back, Int. J. Tuberc. Lung Dis. 8 (2004) 903-904.

[28] R.M. McCune, F.M. Feldmann, H.P. Lambert, W. McDermott, Microbial persistence. I. The capacity of tubercle bacilli to survive sterilization in mouse tissues, J. Exp. Med. 123 (1966) 445-468.

[29] R.M. McCune, F.M. Feldmann, W. McDermott, Microbial persistence. II. Characteristics of the sterile state of tubercle bacilli, J. Exp. Med 123 (1966) 469-486.

[30] D.L. Whipple, C.A. Bolin, J.M. Miller, Distribution of lesions in cattle infected with Mycobacterium bovis, J. Vet. Diagn. Invest. 8 (1996) $351-354$.

[31] D.V. Cousins, L.A. Corner, J.W. Tolson, S.L. Jones, P.R. Wood, in: D.V. Cousins, L.A. Corner (Eds.), Eradication of Bovine Tuberculosis from Australia: Key Management and Technical Aspects, CSL limited, Victoria, Australia, 1998, ISBN 0646347500.

[32] T. Smith, A comparative study of bovine tubercle bacilli and of human bacilli from sputum, J. Exp. Med. 3 (1898) 451-511.

[33] A.S. Griffith, Human tubercle bacilli in the milk of a vaccinated cow, J. Pathol. Bacteriol. 17 (1913) 323-328.

[34] B.E. Willcox, C.R. Willcox, L.G. Dover, G. Besra, Structures and functions of microbial lipid antigens presented by CD1, Curr. Top. Microbiol. Immunol. 314 (2007) 73-110.

[35] I. Van Rhijn, A.P. Koets, J.S. Im, D. Piebes, F. Reddington, G.S. Besra, S.A. Porcelli, W. van Eden, V.P. Rutten, The bovine CD1 family contains group 1 CD1 proteins, but no functional CD1d, J. Immunol. 176 (2006) 4888-4893.

[36] D.B. Moody, T. Ulrichs, W. Muhlecker, D.C. Young, S.S. Gurcha, E. Grant, J.P. Rosat, M.B. Brenner, C.E. Costello, G.S. Besra, S.A. Porcelli, CD1c-mediated T-cell recognition of isoprenoid glycolipids in Mycobacterium tuberculosis infection, Nature 404 (20, 2000) 884-888.

[37] F. Poccia, M. Malkovsky, A. Pollak, V. Colizzi, G. Sireci, A. Salerno, F. Dieli, In vivo gammadelta $\mathrm{T}$ cell priming to mycobacterial antigens by primary Mycobacterium tuberculosis infection and exposure to nonpeptidic ligands, Mol. Med. 5 (1999) 471-476.

[38] A.J. Smyth, M.D. Welsh, R.M. Girvin, J.M. Pollock, In vitro responsiveness of gammadelta $\mathrm{T}$ cells from Mycobacterium bovis-infected cattle to mycobacterial antigens: predominant involvement of $\mathrm{WC1}(+)$ cells, Infect. Immun. 69 (2001) 89-96.

[39] S.G. Rhodes, B.M. Buddle, R.G. Hewinson, H.M. Vordermeier, Bovine tuberculosis: immune responses in the peripheral blood and at the site of active disease, Immunology 99 (2000) 195-202.

[40] M.D. Welsh, H.E. Kennedy, A.J. Smyth, R.M. Girvin, P. Andersen, J.M. Pollock, Responses of bovine WC1(+) gammadelta T cells to protein and nonprotein antigens of Mycobacterium bovis, Infect. Immun. 70 (2002) 6114-6120.

[41] H.E. Kennedy, M.D. Welsh, D.G. Bryson, J.P. Cassidy, F.I. Forster, C.J. Howard, R.A. Collins, J.M. Pollock, Modulation of immune responses to Mycobacterium bovis in cattle depleted of $\mathrm{WC1}(+)$ gamma delta T cells, Infect. Immun. 70 (2002) 1488-1500.

[42] Y. Shen, D. Zhou, L. Qiu, X. Lai, M. Simon, L. Shen, Z. Kou, Q. Wang, L. Jiang, J. Estep, R. Hunt, M. Clagett, P.K. Sehgal, Y. Li, X. Zeng, C.T. Morita, M.B. Brenner, N.L. Letvin, Z.W. Chen, Adaptive immune response of Vgamma2Vdelta2 + T cells during mycobacterial infections, Science 295 (2002) 2255-2258. 